cardiac complications, and the proportion of recoveries. Out of the forty patients, twenty-three were males, and seventeen females.

$$
\begin{aligned}
& \begin{array}{llllllll}
\text { Under } 10 \text { years } & \ldots & \ldots & \ldots & \ldots & \ldots & \ldots & 0
\end{array}
\end{aligned}
$$

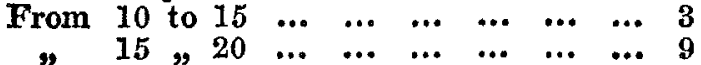

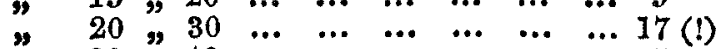

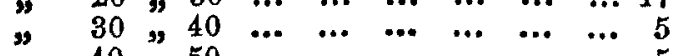

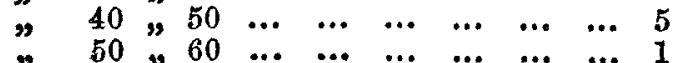

Among the forty cases, twenty-five were affected with cardiac complications:-

$$
\begin{array}{llllllr}
\text { Endocarditis } & \ldots & \ldots & \ldots & \ldots & \ldots & 20 \\
\text { Pericarditis } & \ldots & \ldots & \ldots & \ldots & \ldots & 0 \\
\text { Endo- and peri-carditis } & \ldots & \ldots & \ldots & 5
\end{array}
$$

In three cases of heart affection the lungs were likewise attacked. Of the forty cases, twenty-nine recovered, two died, and nine were, in March 1851, under treatment. We should not omit to note, that one of the two fatal cases was complicated with albuminuria, and the other with endopericarditis and pneumonia, accompanied by a large exomphalos, a small femoral hernia, and an attack of peritonitis. In this latter instance the autopsy showed the pericardium universally adherent, and deposits on the aortic valves.

\section{KING'S COLLEGE HOSPITAL.}

\section{Aneurism on the Common Carotid Artery; Deligation of the Vessel; Death.}

(Under the care of Mr. Ferausson.)

Oon readers may remember that the carotid artery was tied come months ago, with perfect success, by Mr. Johnson, at St. George's Hospital, (The Lancex, July 27, 1850, p. 118.) The deligation of the vessel was here undertaken for the sake of commanding hæemorrhage from the interior of the mouth, brought on by the thrust therein of the point of a parasol. The boy did extremely well, and presented none of the cerebral symptoms which are said sometimes to follow the inter. rupted supply of blood to the brain.

We have now to put upon record an operation of the same kind, performed upon an adult, for the cure of an aneurism of the carotid artery. Though many methods have by turns been proposed to procure the obliteration of the sac,-as presmre, injection, and galvano-puncture, the results have been so unsatisfactory, that the only means likely to afford permanent relief is the deligation of the vessel on the cardiac side of the tumour. Such deligation seems, however, somewhat hazardous, when so important a trunk as the carotid artery is to be tied; but the cerebral disturbance which might naturally be

looked forward to, is in fact very seldom noticed.

It is well known that we are indebted to chance for the discovery of the innocuity of this operation: a surgeon wounds the carotid whilst removing a scirrhous tumour from the side of the face, and to save his patient's life, he ties the vessel, and the case does well. Abernethy was the first, in this country, who attempted the operation; Fleming followed his example, and the deligation of the carotid artery soon became an established and acknowledged operation in surgery. The vessel has been several times tied for the cure of aneurism by mastomosis, or erectile tumours in the cheek or eye: Mr. Travers, Mr. Dalrymple, and Mr. Wardrop, have successfully operated in this country in cases of this nature.

When the deligation is undertaken for the cure of aneurism, it is of course very advantageous that the tumour should be vo situated as to allow of the ligature being applied in that portion of the course of the artery which offers the greatest facility for such operation-viz. in the upper triangle of the neck. But aneurismal tumours are sometimes situated so low down, as to leave but very little room between their lower border and the clavicle; here the dissection will of course be longer, and the whole operation require much caution. Dr. Robertson, of Edinburgh, operated in a case of carotid aneurism where the sac was situated low down, and the tumour had burst into the osophagus; he made his incision to the internal side of the sterno-mastoid muscle only one inch in length, and succeeded in securing the vessel. Mr. Fergusson had to surmount a difficulty of the same kind, as the tumour came close to the clavicle; the artery was, however, laid bare and tied, after a careful dissection, which presented more than nsual difficulty.

Mary $\mathrm{S} \longrightarrow$, aged thirty-eight, and unmarried, was admitted June 11, 1851, under the care of Mr. Fergusson, with a swelling on the left side of the neck, presenting several symptoms of carotid aneurism. The patient has generally enjoyed good health, but has led a dissipated life, and endured great privations. None of her family have suffered from diseased arteries or aneurism. About eighteen months ago she was under treatment at the Westminster Hospital for gangrene of the toes, induced by exposure to wet and cold during two days. and nights. Six months since, the patient began to cough, and to experience difficulty of swallowing; about the same time she likewise noticed a small swelling in the course of the carotid artery on the left side, which, however, gave her no pain. The swelling gradually increased to its present size, and the function of respiration became moreand more impeded. On examination, a tumour, about the size of a small fist, tense, pulsating, and tender to pressure, was noticed on the left side of the neck, over the course of the carotid artery. It extended from the base of the jaw nearly to the upper border of the clavicle. No morbid sound of the heart could be dotected, but a distinct bruit was heard in the tumour; the general arterial system seemed healthy. The breathing was very difficult. On the third day after admission, the patient became very low and weakly; the dyspncea was great, as the tumour seemed to press somewhat against the trachea and larynx. The skin over the swelling was becoming very red and tense.

Mr. Fergusson examined the patient just before proceeding with several other operations; and finding her with so much. difficulty of breathing, and the tumour threatening to burst, he determined to tie the carotid artery on the cardiac side of the tumour. The patient had become suddenly worse, and this circumstance necessitated prompt measures; but it had been Mr. Fergusson's intention to use appropriate internal remedies before undertaking the operation-a precaution which would certainly in many instances be extremely useful.

No delay could, however, intervene in this instance, for there was every probability of the tumour bursting outwardly, as it presented a highly inflamed spot, or the patient dying from suffocation. When she was brought into the theatre, the question arose whether, in her weak state, the patient should inhale chloroform. Mr. Fergusson thought that the anmesthetic agent should be administered, but with great caution.

When the patient was completely insensible, or nearly so, and the head slightly raised, Mr. Fergusson commenced with a longitudinal incision, about three inches long, to the inner side of the mastoid muscle, from the lower portion of the tumour down along the upper end of the sternum. After the cellular tissue, fascia, and platysma had been divided, the sterno-mastoid came into view, and as here the vessels lay very deep, Mr. Fergusson cut this muscle across. The sternohyoid and thyroid were now carefully divided, as well as some loose cellular tissue, and the sheath of the vessels was perceived. This was cautiously opened upon the director, and the aneurism-needle passed round the carotid artery from without inwards, when the vessel was well secured by a strong thread. Temporary tightening caused the pulsations of the tumour to cease, and when this had been ascertained, the ligature was finally fixed, and the lips of the wound brought together by suture.

No cerebral symptom became manifest after the deligation of the artery, but the pulsations of the radials became much slower; the breathing, which had been very laboured, regained some vigour, and the patient was removed in a comparatively satisfactory state.

Mr. Fergusson took occasion to remark, that he had not expected, when the patient was admitted, to operate so soon, as he intended to afford her eight or ten days' rest and appropriate treatment; but the disease had progressed so rapidly, inflammation had set in so quickly, and the dyspnca was so great, that if not interfered with, the tumour would have burst either internally or externally. On consultation with his colleagues he had expressed his conviction, and they had agreed, that the vessel should be tied forthwith, though the space between the clavicle and the lower portion of the tumour was very short. The aneurism was probably situated on the upper part of the common carotid, or just at the bifurcation, but to know the exact position was not important as regarded treatment.

He (Mr. Fergusson) had made his incision very long, reach. ing even over the sternum, as the vessels lie so deep in this locality that plenty of room is very necessary, and on this ground he had divided the sterno-mastoid muscle without hesitation, the more so as this operation is one involving life, and that a muscle was of little importance in comparison. $\mathrm{Mr}$. Fergusson had been somewhat apprehensive of thesmall veinsin that region, in direot communication with the veins of the neck, but the hæmorrhage had altogether been trifling; the jugular 
rein was not seen at all. The best and only step had now been taken for the patient's safety; she might, however, now die from the bursting of the sac either externally or internally. $A$ favourable circumstance in the operation was, that the patient had a rather long neck, and was very thin.

At four o'clock in the afternoon the effects of chloroform had been recovered from, the breathing was more regular than previous to the operation, but there was an accumulation of mucus in the air-passages which the patient conld not force away. She was given squill and hyoscyamus in camphor mixture. At seven in the evening respiration was still less embarrassed, the pulse 90 , the tumour slightly diminished in size, and very tense; but towards three o'clock in the morning the patient was seized with a short convulsive fit, and died soon afterwards, without any previous impairment of cerebral influence.

On a post-mortem examination the parietes of the heart were found very thin and weak; the arterial system immediately above that organ did not exhibit any striking alteration, except the abdominal aorta, where abundant atheromatous deposit was found; the other organs of the chest were healthy. The ligature was firmly fixed, and situated about an inch and a half below the tumour; the latter was filled with coagulum and soft fibrinous masses, and was situated opposite the thyroid body,extending as high as the os hyoides. The aneurism was of the description in which all the coats of the vessels had originally been equally distended; the opening of the artery into the lower part of the sac was smooth and round, and continuous with the walls of the tumour; the upper aperture was about of the same kind, having a slight valve added to it, and the carotid artery, at its emergence, was only half the calibre of the portion which was seen entering the sac. The whole of the latter lay on the left lobe of the thyroid body, on the trachea and cesophagus, these organs being evidently com. pressed by the tumour.

The vagus nerve, imbedded in the walls of the sac, was seen entering and emerging from these walls, close to the artery; in gently pulling the nerve above and below, its outline could be seen tightening along the parietes of the sac. This peculiar disposition of the vagus may be looked upon as greatly conducive to the intense dyspnoea which the patient auffered for the last week of her life.

The diagnosis of this case did not offer difficulties of a serious nature, but it will be conceded that too much care cannot be bestowed upon the recognition of the actual nature of the disease; for it is principally in the neck that tumours and abscess may simulate aneurism. Enlarged glands are especially liable to create deception; but it is seldom that the thrill communicated to the hand, or the bruit conveyed to the ear, are so distinct as in true aneurism, as was observable in the foregoing case. The full dilatation of all the coats of the vossels took place in a comparatively short time, six months only having elapsed since the patient perceived the swelling; for such cases as the one related by Mr. Hodgson, where a femoral aneurism destroyed an old man in three weeks, are certainly very rare. It will be perceived that Mr. Fergusson's patient was a female: we need not point out how seldom aneurisms affect women, this fact lending, as we venture to think, much weight to the supposition that violent exertion has much influence on the formation of aneurismal tumours, where vessels are not in a sound state.

\section{Sthedical Eocíties.}

ROYAL MEDICAL AND CHIRURGICAL SOCIETY. Tuesday, Jung 24, 1851.-Mr. Hodgson, Pregident.

On some Secondart Phystologican Fifects produced bx Atmospheric Electrictix. By Professor C. F. Bchonbein. AFTER referring to the more obvious effects of electricity upon the organs of sensation, and more especially those of smell and taste, and stating his belief that the peculiar odour observed when an electrical discharge takes place, or in the neighbourhood of points from which electricity is passing, is not due to the electricity itself, but caused by the presence of a peculiar matter which he has named ozone, the author proceeded to lay before us the opinions held respecting it-viz, that either pure or atmospheric oxygen, when exposed to the action of electricity, is transformed into this odoriferous body, which he was at first inclined to consider a peculiar peroxide of hydrogen, bat which other eminent chemists believe to be merely an allotropic modification of oxygen. With reference to its exact composition he declines, however, to give a decided opinion. This body, whatever its actual nature may be, is a most powerful oxidizing agent; at ordinary temperatures, oxidizing silver, forming iodic acid from iodine, nitric acid from nitrogen; converting the acids ending in ous into those which end in ic, the salts which terminate in ite into those which end in ate; metallic sulphurets into sulphates. It decomposes the gaseous components of hydrogen, with sulphur, selenium, phosphorus, iodine, arsenic, and antimony, and exerts many other energetic chemical actions, both on organic and inorganic substances. It has a strong electro-motive power, similar to that of chlorine, bromine, and iodine; and lastly, it has certain physiological effects upon the animal system, similar to those of chlorine and bromine, acting powerfully as a poison, even in minute doses. Ozone is being constantly generated in the atmosphere by the electrical action going on. When in excess it has a sensible effect upon the mucous membranes of those exposed to its influence, and its presence is indicated by starch containing the iodide of potassium being turned blue. The author has availed himself of this fact in the construction of his ozonometer, which consists of a box containing testpaper thus prepared, and a chromatic scale. He believes that certain catarrhal affections are produced by the presence of ozone in the atmosphere, and he has availed himself of the assistance of medical friends in ascertaining the co.existence of unusual prevalence of catarrh with what he terms his blue days, and he suggests that more extended observations are desirable for this purpose. The author then referred to the existence of poisonous miasmata in the atmosphere, which are generated by certain " purely chemical, or physical, or physiologicaliy chemical actions," which take place within the earth, or on its surface, in stagnant or running waters, or in the atmosphere itself. Of these poisonous gases or vapours, there are two sorts which are antagonistic to and destroy each other. Of the first class, sulphuretted, and perhaps phospuretted, hydrogen, alone, are produced by natural causes, and these in such minute quantities that they do not, except in a fer localities, exert any general influence upon animal life. OE the second class, there is one, namely ozone, which exists in appreciable quantities in the atmosphere. But the abundant source of gaseous matters is that which the decomposition of vegetable and animal substances affords. Some of these are well known, such as carbonic acid and ammonia; others are of unknown chemical nature, and though the absolute quantit of such deleterious matters may be small in comparison to the immense volume of the atmosphere, their accumulation would render the air unfit for the support of animal life, unlese some agent were at work to neutralize or decompose them. The author states, that the purification of the atmosphere which we attempt to effect on a small scale by chlorine fumigations, is accomplished in the great processes of nature by the agency of ozone, a task for which it is peculiarly fitted by its high oxidizing powers. He gives the details of some experiments upon air tainted by putrid flesh, which was purified by ozone disengaged by the action of moistened phos* phorus. Ozone, which is formed in the atmosphere by the electrical discharges constantly going on, acts upon and de. composes the oxidizable miasmatic gaseous matters which contaminate it, and thus the atmosphere is preserved in a state fit for the support of animal life, and, at the same time, the ozone, which, if allowed to accumulate, would become deletorious, is in its turn neutralized or decomposed by the miasmata. During a thunder-storm large quantities of azone are formed, and the author supposes that the unhealthy state of the atmo sphere which exists in the hot season of the year may be caused by the accumulation of miasmata produced by animal decomposition; and that it is by the generation of ozone that thunderstorms purify the air. The author considers that there are probably certain states of the atmosphere in which the quantity of ozone does not bear a due propor. tion to the miasmata it has to act upon, and that under suoh circumstances it is that certain diseases-cholera, for instance -make their appearance. He is also of opinion, that in the winter there is more atmospheric ozone than in summer, and that the higher strata of the atmosphere contain more than the lower; and as the generation of some diseases, such as the yellow fever, appears to be connected with certain seasong and geographical positions, he thinks that by an extended series of observations it might be ascertained whether these diseases bear any relation to the ozoniferous state of that portion of the atmosphere where they happen to occur. The paper concludes with a reference to experiments which prove that ozone produced by the action of electricity or by the ageney of phosphorus is identical. 